\title{
A essencialidade das academias de ginástica para a saúde diante da pandemia da COVID-19 no Brasil
}

\section{The essentiality of gyms for health in the face of the COVID-19 Pandemic in Brazil}

\section{AUTORES \\ Fabio Fortunato Brasil de Carvalho ${ }^{1}$ (i) Rodolfo Guimarães da Silva ${ }^{2,3,4}$ (iD \\ Ricardo Brandão Oliveira ${ }^{3,4}$ (DD \\ 1 Associação Brasileira de Saúde Coletiva, Rio de Janeiro, Rio de Janeiro, Brasil. \\ 2 Centro Universitário de Volta Redonda, Volta Redonda, Rio de Janeiro, Brasil \\ 3 Programa de Pós Graduação em Ciências do Exercício e do Esporte, Universidade do Estado do Rio de Janeiro, Rio de Janeiro, Brasil. \\ 4 Laboratório de Vida Ativa. Universidade do Estado do Rio de Janeiro. Rio de Janeiro, Brasil.}

\section{CONTATO}

Ricardo Brandão Oliveira ricardobrandaorj@gmail.com

Rua São Francisco Xavier 524, Maracanã, Rio de Janeiro, Rio de Janeiro, Brasil. CEP: 20550-900.

DOI

10.12820/rbafs. $25 \mathrm{e} 0116$

\section{(cc) BY}

Este trabalho está licenciado com uma Licença Creative Commons - Atribuição 4.0 Internacional.

\begin{abstract}
RESUMO
Em maio de 2020, por decreto presidencial, as academias foram incluídas no rol de atividades essenciais. Embora o decreto presidencial não tenha sido adotado por muitos estados e municípios, pressões e posicionamentos em favor da reabertura do setor têm ocorrido. Este ensaio discutiu a essencialidade destes serviços e propôs elementos para pautar esta decisão, tomando como base uma sucinta análise de benefícios e riscos sob a ótica individual e coletiva por meio de pesquisa exploratória. Os diversos efeitos positivos da atividade física sobre a saúde permitiriam pressupor a importância da reabertura de academias de ginástica, contudo há distinções entre benefícios e riscos a curto e longo prazos relacionados às doenças crônicas e ao COVID-19 que permitem afirmar que as atividades físicas em âmbito domiciliar são mais adequadas neste momento da pandemia. Assim, não é momento de reabertura de academias de ginástica no país, sobretudo, por não as considerarmos como atividades essenciais.
\end{abstract}

Palavras-chave: Quarentena; Atividade motora; Mortalidade; Política pública.

\section{ABSTRACT}

In May 2020, by presidential decree, gym centers were included in the list of essential activities. Although many states and municipalities did not adopt the presidential decree, it provided pressures in favor of the reopening. In the present study, we discuss the essentiality of these services and propose elements to guide this decision, based on a succinct analysis of benefits and risks from an individual and collective perspective through exploratory methods. The evidence on the various positive effects of physical activity on health is robust, which would allow us to assume the importance of reopening fitness centers. However, there are distinctions between short and long term benefits and risks related to chronic diseases and COVID-19 that allow us to affirm that physical activities at home are more appropriate for this moment of the pandemic in which there is an increasing number of infected and deaths.

Keywords: Quarentine; Motor activity; Mortality; Public policy.

\section{Introdução}

No dia 8 de maio de 2020, por decreto presidencial $^{1}$, as academias de esportes (e de ginástica) de todas as modalidades foram incluídas no rol de atividades essenciais (classificadas como indispensáveis e inadiáveis na medida em colocam em perigo a sobrevivência, a saúde ou a segurança da população). Este decreto foi de encontro ao então posicionamento do Ministério da Saúde, fato que ganhou notoriedade pública.

Ainda que o governo federal tenha estabelecido atividades essenciais em meio à pandemia, o Supremo Tribunal Federal (STF) apontou que caberia aos estados e municípios o poder de estabelecer políticas de saúde - inclusive sobre as questões de distanciamento social e serviços considerados essenciais. Muito embora o decreto presidencial não tenha sido adotado por muitos estados e municípios, diferentes instituições passaram a exercer pressões e a publicar notas de protestos em favor da reabertura das academias de ginástica.

A partir deste cenário e considerando os impactos da atividade física sobre aspectos sociais, econômicos e da saúde, seja sob a ótica fisiológica ou clínico-epidemiológica $^{2}$, com destaque, neste momento da pandemia, para seus efeitos sobre o sistema imunológico e sobre a saúde mental, o objetivo deste ensaio é discutir a essencialidade das academias de ginástica e propor elementos para pautar esta decisão, tomando como base uma sucinta análise de benefícios e riscos sob a ótica individual e coletiva. 


\section{Essencialidade das academias de ginástica - ponderações diante de uma pandemia}

Para iniciar essa reflexão, considerações sobre os impactos imediatos, de médio e de longo prazo sobre a saúde devido à pandemia da COVID-19 são necessárias. Diferentes curvas de impactos sobre a saúde são previstas ${ }^{3}$. A primeira e mais importante a ser considerada refere-se ao impacto imediato sobre a mortalidade, atualmente com uma taxa de letalidade no Brasil de 5,2\%, segundo dados da Universidade de Johns Hopkins atualizados em 09 de junho de $2020^{4}$.

Por maiores que sejam os efeitos fisiológicos de curto prazo da inatividade física, estes não se traduzem necessariamente por desfechos clínicos imediatos. Diferentemente do que é observado para grupos populacionais de alto risco, como no caso de pacientes com insuficiência cardíaca grave, que podem apresentar taxas de sobrevida de $47 \%$ e $32 \%$ em 1 e 2 anos, respectivamente ${ }^{5}$, grupos populacionais mais saudáveis costumam apresentar desfechos clínicos de maior prazo, dado o lento curso natural da maioria das doenças crônicas não transmissíveis (DCNTs).

Neste sentido, Blair et al. ${ }^{6}$, ao avaliar 9.777 homens saudáveis demonstraram curvas de sobrevida sem diferenças significativas no curto prazo, entre os grupos que mantiveram a baixa aptidão física (unfit - unfit) e aqueles que foram capazes de aumentar sua aptidão física (unfit-fit) ao longo dos anos do estudo.

Com base nesses achados e partindo do pressuposto teórico que o distanciamento social imposto pela $\mathrm{CO}$ VID-19 tenha aumentado significativamente o nível de inatividade física da população brasileira, apesar da escassez de dados, não seria de se esperar um aumento significativo no risco de mortalidade em curto prazo de grupos populacionais mais saudáveis.

Diferentemente das DCNTs, que apresentam curso natural lento, a COVID-19, com uma alta taxa de contágio e de letalidade no Brasil de 5,2\% ${ }^{4}$, apresenta um curso rápido até o óbito, especialmente entre os pacientes que manifestam sintomas graves da doença, com tempo de internação longo o suficiente para sobrecarregar os sistemas de saúde, levando-os muitas vezes ao colapso ${ }^{7}$.

Neste sentido, considerando a elevada curva de mortalidade associada à COVID-19 e o baixo risco de mortalidade em curto prazo de grupos populacionais mais saudáveis, acreditamos que a reabertura de academias não representaria, já por esta razão, ganho significativo, em uma perspectiva de saúde pública, diante do cenário atual da pandemia da COVID-19.

Assim, dois novos questionamentos são necessários ao debate proposto neste artigo. $\mathrm{O}$ primeiro, seria o papel das atividades físicas domiciliares na mitigação dos impactos da inatividade física da população. $\mathrm{O}$ segundo, o papel das academias de ginástica nesse contexto.

\section{Atividades físicas domiciliares versus academias de ginástica - quais seus papéis na mitigação dos impactos sobre os níveis de atividade física da população?}

Não iremos nos alongar sobre os efeitos das atividades físicas domiciliares, em especial no contexto da pandemia da COVID-19, uma vez que o tema já foi bem explorado, apresentando os diversos benefícios das atividades físicas domiciliares e demonstrando ser de grande viabilidade, segurança e eficácia na prevenção primária e secundária de doenças e eventos cardiovasculares, sendo, portanto, uma importante estratégia de intervenção que deveria nortear políticas de mitigação dos impactos da pandemia sobre os níveis de inatividade física ${ }^{8}$.

Passemos agora ao papel das academias de ginástica nesse contexto. A primeira questão que nos provoca é saber o percentual de brasileiros que costumavam frequentar academias de ginástica em períodos anteriores ao isolamento social imposto pela maioria dos Estados no país. Em 2017, o Brasil era o quarto país do mundo com o maior número de clientes no setor, totalizando 9,6 milhões, o que representaria naquele ano, aproximadamente $4,5 \%$ da população brasileira.

De imediato, tal fato já nos revelaria a importância do fortalecimento de políticas públicas voltadas à promoção da atividade física no país que, devido a sua desigualdade estrutural, não permite que parte importante de sua população tenha acesso a locais privados para a prática de atividade física supervisionada por profissionais.

No entanto, diante do cenário atual da pandemia, soma-se, ainda, um importante argumento que vai de encontro a essencialidade desses serviços. Mesmo com a reabertura de academias, seja a partir do decreto presidencial e/ou de ações normativas de governos ou poder legislativo estaduais e municipais, há a recomendação ou estímulo para que grupos de risco, que incluem idosos e aqueles com comorbidades associadas ao agravamento da COVID-19, não frequentem as academias neste momento, permanecendo em distanciamento social. 
Segundo dados do projeto Monitora COVID-19, coordenado pela Fundação Oswaldo Cruz (FIOCRUZ), estima-se que um em cada três brasileiros $(33,5 \%)$ acima de 18 anos tenham ao menos um dos principais fatores associados a complicações da COVID-19: hipertensão, diabetes, doenças do coração ou do pulmão? .

Assim, nos parece claro, diante das evidências, que uma vez reabertas as academias de ginástica, restariam, dentre seus frequentadores, um percentual diminuto da população brasileira, composto eminentemente pelos mais jovens e saudáveis, grupo populacional de menor risco de mortalidade associados às DCNTs.

Em adendo, dados sugerem que indivíduos assintomáticos possam ser responsáveis por transmissões de $46 \%$ a $62 \%$ dos casos de transmissão do vírus, fato que também coloca em questionamento a reabertura das academias eminentemente ao público jovem, sem fatores de risco e sem sintomas ${ }^{10}$.

\section{Cenário atual da Pandemia de COVID-19 e medidas de enfrentamento}

Dados publicados em 25 de maio de 2020, pelo projeto EPICOVID19, sugerem que a pandemia da COVID-19 no Brasil ainda está em sua fase inicial, uma vez que apenas $1,4 \%$ de brasileiros tiveram contato com o vírus no país ${ }^{11}$.

Caso houvesse o relaxamento das medidas de distanciamento social a partir da referida data, a multiplicação no número de casos provocaria uma nova curva com milhares de novos óbitos. Em adendo, foi estimado que o número de casos na população seja sete vezes superior ao apresentado nas estatísticas oficiais. Isso representa que de cada 7 pessoas infectadas, apenas uma sabe que está ou esteve infectada e que as demais 6 pessoas que não sabem da infecção podem, involuntariamente, transmitir o vírus para outras pessoas ${ }^{11}$.

Enquanto os países trabalham para encontrar o equilíbrio entre as consequências para a saúde e as perturbações econômicas causadas pela COVID-19, a dicotomia "sociedade aberta" versus "fechada" deverá ser desconstruída. Os níveis de risco podem subir e descer, dependendo da expansão, contenção da disseminação do vírus, e outros fatores, por longos meses.

Os governos precisarão trabalhar com as comunidades para comunicar os níveis variados de gravidade e risco de surtos e ajudar as pessoas a adotar comportamentos de proteção enquanto tomadores de decisão implementam as mudanças políticas necessárias para limitar o impacto da pandemia. Dados sugerem que distanciamentos sociais intermitentes deverão ser necessários até $2022^{12}$, enquanto que a Organização Mundial da Saúde (OMS) recomenda que medidas de controle precisarão ser continuamente implementadas e monitoradas ${ }^{13}$, a saber:

1) transmissão do vírus controlada, normalmente expressa pelo número básico de reprodução (R0) inferior ao valor zero. Apesar de observarmos, desde o início das medidas de distanciamento social em 23 de março, uma redução da taxa de reprodução no Brasil, o valor médio no país ainda é de 1,4 (variando entre 1,08 a 5,63) ${ }^{14}$;

2) sistema de saúde com capacidade de testar, tratar e isolar todas as pessoas com COVID-19 e seus contatos mais próximos. Segundo a OMS, 10 a 30 testes deveriam ser realizados para cada novo caso confirmado. No entanto, segundo fontes das secretarias estaduais de saúde, até o dia 9 de junho de 2020, haviam sido realizados, no Brasil, apenas 990.351 testes. Considerando que nesta data haviam 711.696 casos confirmados no país, o número de testes deveria estar aproximadamente entre 7 e 21 milhões;

3) controlar surtos em locais específicos, como as próprias instalações hospitalares, com a oferta de infraestrutura e recursos humanos adequadas à realidade local. É público e notória a limitação de leitos e infraestrutura disponível no SUS em muitos municípios brasileiros.

4) medidas preventivas de controle e monitoramento em ambientes de trabalho, transporte, escolas, universidades, etc., evitando novos pontos de contágio;

5) controle adequado de possíveis novos casos importados;

6) comunidade informada e engajada nas medidas de higiene e novas normas, conquistadas através de um discurso claro e coeso dos governos municipais, estaduais e federal.

Cabe destacar que tais medidas podem e devem ser pensadas não apenas em âmbito nacional, mas também localmente, norteando, assim, decisores políticos ao nível municipal quanto ao relaxamento ou retomada de medidas de distanciamento social e reabertura de serviços, incluindo, nesse contexto, as academias de ginástica. Em adendo, fica claro que o Brasil apresenta grandes desafios para superar a atual pandemia e que tais medidas precisam ser implementadas com urgência. 


\section{Considerações finais}

É de fundamental importância esclarecer que, como profissionais de Educação Física, defendemos a importância da atividade física a partir de seus benefícios fisiológicos, clínico-epidemiológicos, econômicos e sociais e que também reconhecemos o valor econômico do setor, inclusive sob a ótica do profissional de educação física. Não nos atreveremos aqui, no entanto, a aprofundar discussões sobre os impactos econômicos associados a reabertura das academias e afrouxamento do distanciamento social neste momento da pandemia, mas convidamos os colegas pesquisadores especialistas a fazerem em futuros estudos.

Valorizamos a iniciativa de algumas empresas do setor, bem como a de alguns conselhos regionais de Educação Física, de iniciar o planejamento para a reabertura gradual das academias, mas, com base nas evidências aqui apresentadas, recomendamos que esta reabertura não ocorra neste momento da pandemia. Esperamos que, em um cenário de pós-pandemia, a promoção de atividade física seja ampliada com a implementação de políticas públicas intersetoriais, como a ampliação de programas públicos, a existência de incentivos financeiros associados ao uso de transportes ativos, contribuindo, assim, para a redução do número de carros em centros urbanos, dentre outras.

Por fim, destacamos que 60\% dos brasileiros, nos dias 25 e 26 de maio de 2020, apoiaram que os governos em seus Estados implementem o Lockdown ${ }^{15}$. Os motivos pelos quais os entrevistados fizeram essas escolhas não foram avaliados, mas podemos supor que ainda exista um grande sentimento de insegurança por parcela importante da população.

Entendemos, portanto, o distanciamento social não como um dever do cidadão, mas sim como um direito à vida assegurado na Constituição Federal, que afirma ser objetivo fundamental da República Federativa do Brasil, "promover o bem de todos, sem preconceitos de origem, raça, sexo, cor, idade e quaisquer outras formas de discriminação".

\section{Conflitos de interesse}

Os autores declaram não haver conflito de interesses.

\section{Contribuições dos autores}

Carvalho FFB, Silva RG e Oliveira RB foram igualmente responsáveis pela concepção, análise e interpretação dos dados, redação do artigo e revisão crítica relevante do conteúdo intelectual, aprovação final da versão a ser publicada e por todos os aspectos do trabalho na garantia da exatidão e integridade de qualquer parte da obra.

\section{Referências}

1. Brasil. Presidência da República. Secretaria-Geral. Subchefia para Assuntos Jurídicos. Decreto No 10.344, de 8 de maio de 2020. Altera o Decreto $n^{\circ} 10.282$, de 20 de março de 2020, que regulamenta a Lei $n^{\circ} 13.979$, de 6 de fevereiro de 2020, para definir os serviços públicos e as atividades essenciais. 2020.

2. Gabriel BM, Zierath JR. The Limits of Exercise Physiology: From Performance to Health. Cell Metab. 2017; 25(5):1000-11.

3. Heale R. Nursing and Post Pandemic Health Challenges. Blog: Evidence-Based Nursing. [citado 2020 abr 15]. Disponível em: https://blogs.bmj.com/ebn/2020/04/15/ nursing-and-post-pandemic-health-challenges/.

4. Johns Hopkins University. Coronavirus Resource Center. [citado 2020 jun 9). Disponível em: https://coronavirus.jhu. edu/data/mortality.

5. Mancini DM, Eisen H, Kussmaul W, Mull R, Edmunds Junior L, Wilson J. Value of peak exercise oxygen consumption for optimal timing of cardiac transplantation in ambulatory patients with heart failure. Circulation. 1991;83(3):778-86.

6. Blair SN, Kohl HW 3rd, Barlow CE, Paffenbarger RS Jr, Gibbons LW, Macera CA. Changes in physical fitness and all-cause mortality. A prospective study of healthy and unhealthy men. JAMA. 1995;273(14):1093-8.

7. Canabarro A, Tenorio E, Martins R, Martins L, Brito S, Chaves R. Data-Driven Study of the COVID-19 Pandemic via Age-Structured Modelling and Prediction of the Health System Failure in Brazil amid Diverse Intervention Strategies 2020. medRxiv. 2020.04.03.20052498.

8. Peçanha T, Goessler KF, Roschel H, Gualano B. Social isolation during the covid-19 pandemic can increase physical inactivity and the global burden of cardiovascular disease. Am J Physiol Heart Circ Physiol. 2020;318:H1441-6.

9. Fundação Oswaldo Cruz - Fiocruz. Monitora Covid-19. [Acessado em 2020 jun 01). Disponível em: https://bigdatacovid19.icict.fiocruz.br/.

10. Li R, Pei S, Chen B, Song Y, Zhang T, Yang W, et al. Substantial undocumented infection facilitates the rapid dissemination of novel coronavirus (SARS-CoV-2). Science. 2020;368(6490):489-93.

11. Epicovid 19. COVID-19 no Brasil: várias epidemias num só país Primeira fase do EPICOVID19 reforça preocupação com a região Norte. [Acessado em 2020 mai 27] Disponível em: http://epidemio-ufpel.org.br/uploads/ downloads/276e0cffc2783c68f57b70920fd2acfb.pdf.

12. Kissler SM, Tedijanto C, Goldstein E, Grad YH, Lipsitch M. Projecting the transmission dynamics of SARSCoV-2 through the postpandemic period. Science. 2020;368(6493):860-8.

13. World Health Organization. Critical preparedness, readiness and response actions for COVID-19. [Acessado em 2020 Jun 9]. Disponível em: https://www.who.int/publications-detail/criticalpreparedness-readiness-and-response-actions-for-covid-19.

14. O Globo. Taxa de contágio da Covid-19 mostra crescimento em estados ainda pouco atingidos, e Rio e SP longe da estabilização. [Acessado em 2020 jun 10]. Disponível em: https://oglobo. globo.com/sociedade/coronavirus/taxa-de-contagio-dacovid-19-mostra-crescimento-em-estados-ainda-poucoatingidos-rio-sp-longe-da-estabilizacao-24449549 
15. Datafolha Instituto de Pesquisa. Opinião sobre lockdown. Isolamento Social. Maio de 2020. [Acessado em 2020 mai

Recebido: 03/06/2020

29]. Disponível em: http://datafolha.folha.uol.com.br/ Aprovado: 02/07/2020 opiniaopublica/2020/05/1988729-60-sao-favoraveis-afechamento-total-para-conter-coronavirus.shtml

\section{Como citar este artigo:}

Carvalho FFB, Silva RG, Oliveira RB. A essencialidade das academias de ginástica para a saúde diante da pandemia da COVID-19 no Brasil. Rev Bras Ativ Fis Saúde. 2020;25:e0116. DOI: 10.12820/rbafs.25e0116 